\title{
Lipophilisation de composés phénoliques par voie enzymatique et propriétés antioxydantes des molécules lipophilisées
}

\author{
Luis Javier LOPEZ-GIRALDO ${ }^{1}$ \\ Mickaël LAGUERRE ${ }^{1}$ \\ Jérôme LECOMTE ${ }^{1}$ \\ Maria-Cruz FIGUEROA-ESPINOZA ${ }^{1,2}$ \\ Michel PINA ${ }^{1}$ \\ Pierre VILLENEUVE ${ }^{1}$ \\ ${ }^{1}$ UMR IATE Laboratoire de Lipotechnie, \\ CIRAD, TA B-62/16, \\ 73 avenue Jean François Breton, \\ 34398 Montpellier cedex 5, France \\ <villeneuve@cirad.fr> \\ 2 IRC Montpellier Sup Agro, \\ 1101 Av. Agropolis-CS24501, \\ 34093 Montpellier cedex 5, France
}

Article reçu le 12 novembre 2006

accepté le 12 décembre 2006

\begin{abstract}
The oxidative modification of lipids in foods and cosmetics may lead to alteration of their qualities and can have physiopathological repercussion on consumer. To counteract these inauspicious effects, phenolic compounds, derived from plants, such as phenolic acids and flavonoids seem to be particularly promising. However, their relatively low solubility in apolar media limits their application in oil-based products. In emulsion, thanks to their water-solubility, these compounds are located in aqueous compartment, whereas lipid peroxidation occurs at the oil-water interface. Therefore, there is an interest in trying to enhance the amphiphilic character of phenolic antioxidants by enzymatic lipophilization which corresponds to the grafting of an aliphatic chain (fatty alcohol, acid or amine) through lipase-catalysed esterification or amidation reactions. Due to its amphiphilic nature, the resulting functionalized antioxidant would accumulate at the oil-water interface, increasing protection of target-lipids from oxidation. Various operational conditions and strategies can be adopted to perform lipase-mediated hydrophobation with high yields. This paper firstly presents a panorama of the advances in this field. Finally, the antioxidant capacity of lipophilized compounds is reviewed and compared with that of the native molecule.
\end{abstract}

Key words: phenolic compounds, lipase, lipophilization, esterification, antioxidant capacity

\section{Introduction}

L'oxydation constitue probablement l'un des paramètres majeurs à l'origine de l'altération des produits alimentaires et cosmétiques. Les dégradations oxydatives qui en résultent affectent les qualités nutritionnelles et sensorielles des produits et peuvent avoir des répercussions sur la santé du consommateur. Dans ce contexte, différents moyens de prévention sont disponibles pour limiter ces phénomènes. Parmi eux, la valorisation d'antioxydants d'origine végétale à des fins alimentaires, cosmétiques ou pharmaceutiques représente un enjeu majeur pour la recherche et I'industrie. En effet, leur utilisation en tant que conservateurs naturels pourrait constituer une alternative attractive aux antioxydants synthétiques qui souffrent d'une moindre acceptation par le consommateur.

Parmi les antioxydants naturels, les composés phénoliques, et plus particulièrement les acides phénoliques et les flavonoïdes, suscitent un intérêt grandissant. Cependant, la mise en œuvre de ces molécules polaires dans les matrices lipidiques ou les formulations de type émulsions est délicate et peut s'accompagner d'une diminution de leur efficacité à protéger les lipides contre l'oxydation. Pour contourner ce problème, l'une des stratégies consiste à diminuer considérablement la polarité de ces molécules par le greffage de chaînes aliphatiques grasses, afin d'ajuster leur positionnement dans les matrices alimentaires ou cosmétiques. Le résultat attendu est une facilité $d^{\prime}$ incorporation accrue et une amélioration significative de leur action antioxydante. Il est en effet postulé que de telles modifications de la balance hydrophile/lipophile permettraient d'obtenir de nouvelles molécules bioactives dont les propriétés biologiques seraient améliorées par rapport aux molécules initiales.

Ces réactions, dites de lipophilisation, sont en général effectuées par des procédés biocatalysés mettant en œuvre plus particulièrement des lipases (E.C. 3.1.1.3. triacylglycérol ester hydrolases). Ces dernières sont utilisées dans différents domaines comme l'industrie des détergents (nettoyage des taches de gras, traitements des eaux usées), du papier (hydrolyse des résines pouvant s'accumuler sur les équipements et entraîner une détérioration du papier) et du cuir (dégraissage des peaux, remplacement des solvants organiques). Les lipases sont aussi largement employées dans les domaines des arômes et de la chimie fine, dans l'industrie pharmaceutique pour la résolution de mélanges racémiques et finalement, dans I'industrie des huiles et graisses alimentaires où ces enzymes ont démontré qu'elles étaient une alternative aux catalyseurs chimiques pour la production de nouveaux produits aux propriétés physico-chimiques et aux qualités nutritionnelles améliorées (biofaçonnement des huiles et corps gras). Leur principal avantage, par rapport aux catalyseurs chimiques classiques, réside dans leur plus grande spécificité vis-à-vis du substrat et leur mise en œuvre dans des conditions réactionnelles modérées (température, pression). De plus, le maintien de leur activité dans les solvants organiques élargit considérablement leur champ d'application [1]. Concernant le biofaçonnement des corps gras, il s'agit d'opérations de restruc- 
turation de ces matières premières, pour lesquelles la régiosélectivité ou la typosélectivité (préférence pour un type d'acides gras particulier) rend possible la production de triacylglycérols spécifiques présentant une composition et une distribution en acides gras prédéterminée. Ainsi, les lipases sont utilisées dans la production d'équivalents de beurre de cacao [2], de graisses à basses calories [3] ou de substituts lipidiques de lait maternel [4]. Des procédés similaires ont également été décrits pour la synthèse de glycérides partiels ou de concentrés d'acides gras. Un grand nombre de revues a d'ailleurs été publié [5-9]. Enfin, dans le secteur alimentaire, ces enzymes sont employées pour la synthèse d'émulsifiants de type esters de glucides, connus sous le vocable de sucro-esters. Dans cet article seront décrits et discutés plus particulièrement certains des résultats récents publiés sur l'utilisation spécifique des lipases pour lipophiliser des molécules bioactives telles que les dérivés phénoliques. Nous détaillerons également les nouvelles propriétés antioxydantes de ces composés obtenus par modulation de leur balance hydrophile/lipophile.

\section{Stratégies de synthèse pour les réactions de lipophilisation biocatalysée par des lipases}

La lipophilisation enzymatique telle que décrite dans cet article correspond au greffage d'une chaîne grasse (acide, amine ou alcool gras) sur une molécule hydrophile de type sucre, acide aminé ou dérivé phénolique. Dans ce type de réactions, cependant, l'association de deux substrats de polarité très différentes (lipophile/hydrophile) constitue la principale difficulté à surmonter pour garantir une cinétique et un rendement satisfaisants lors de la biocatalyse [10]. Plusieurs paramètres clés doivent alors être considérés. En tout premier lieu, la nature du milieu organique choisi est primordiale. Bien entendu, la stratégie la plus intéressante consiste à réaliser la réaction en milieu fondu, c'est-à-dire en absence de solvant. Cependant, lorsque cela n'est pas possible, le choix d'un solvant adéquat s'avère crucial. Ce dernier doit être inerte vis-à-vis de l'enzyme et ne pas altérer son activité. Ceci est généralement le cas pour les solvants possédant une valeur de log $\mathrm{P}$ supérieure à 3 , comme I'hexane ou I'heptane par exemple (tableau 1); la valeur de log P correspondant au coefficient de partition d'un solvant entre l'eau et l'octanol. Ces solvants sont couramment utilisés dans des réactions de type biofaçonnement des huiles et des graisses, catalysées par des lipases. Or, de manière générale, il n'existe pas de milieu approprié aux réactions qui mettent en jeu des substrats dont les polarités diffèrent radicalement. Le meilleur exemple pour illustrer cette difficulté est probablement la synthèse enzymatique de sucro-esters aliphatiques pour laquelle une molécule hydrophile (glucide) doit être combinée à une molécule lipophile (acide gras). Dans ce cas, les solvants possédant une valeur de log $\mathrm{P}$ supérieure à 3 ne conviennent pas dans la mesure où la solubilité du glucide dans ce milieu est très faible, voire nulle. Les solvants avec de faibles valeurs de $\log P$ peuvent être employés et plusieurs exemples sont trouvés dans la littérature avec l'acétonitrile [11, 12], le tert-butanol [13, 14], le 2-méthyl-2butanol [15-17] et l'éthylméthyl cétone [18]. Un bon contact entre les

Tableau 1. Valeurs de $\log P$ de différents solvants utilisés dans les réactions par catalyse lipasique.

\begin{tabular}{|llcc|}
\hline Solvant & $\log \mathbf{P}$ & Solvant & $\log \mathbf{P}$ \\
\hline Heptane & 4 & Tétrahydrofurane & 0,5 \\
Hexane & 3,5 & Acétone & $-0,3$ \\
Toluène & 2,5 & Acétonitrile & $-0,4$ \\
Benzène & 2 & Éthylméthyl cétone & $-0,8$ \\
2-Méthyl-2-butanol & 1,3 & Diméthylformamide & -1 \\
tert-Butanol & 0,8 & Dioxane & $-1,1$ \\
Pyridine & 0,7 & Dimétylsulfoxide & $-1,3$ \\
\hline
\end{tabular}

deux substrats doit être recherché et le solvant sélectionné doit pouvoir les solubiliser, tout du moins partiellement. C'est pourquoi les milieux présentant une polarité intermédiaire sont fréquemment choisis. À cet égard, les alcools tertiaires sont de bons candidats, notamment parce qu'ils ne sont pas compétitifs des substrats mis en œuvre vis-à-vis des lipases. Qui plus est, leur élimination en fin de réaction n'est généralement pas problématique. L'autre paramètre important dans ce type de réaction concerne l'enzyme elle-même, et plus particulièrement son conditionnement. Durant ces vingt dernières années, les différentes techniques de conditionnement des lipases ont grandement amélioré le champ de l'ingénierie moléculaire et permis leur immobilisation sur un support inerte. Dans ce cadre, l'objectif est d'améliorer certaines de leurs propriétés, telles que l'activité catalytique, la spécificité vis-à-vis du substrat, la stabilité ( $\mathrm{pH}$ ou gamme de température) ou l'aptitude à être recyclées.

Ceci étant, le paramètre le plus important à maîtriser en synthèse enzymatique est sans conteste le micro-environnement aqueux de I'enzyme qui se traduit par l'activité thermodynamique de l'eau $\left(a_{w}\right)$. La conduite de réactions d'estérification et de transfert d'acyles en phase aqueuse limitée, telles que l'interestérification ou la transestérification biocatalysées par les lipases, requiert l'étude préalable de l'influence de $l^{\prime} a_{w}$ de l'enzyme sur la cinétique et le rendement de la réaction. En effet, ce paramètre détermine la quantité d'eau liée à l'enzyme qui lui est nécessaire pour assurer sa structure active optimale. A contrario, un excès d'eau dans la catalyse lipasique donnerait lieu à des réactions d'hydrolyse compétitives. Il est donc primordial de maîtriser l'a $a_{w}$ et en conséquence la teneur en eau pour définir les conditions réactionnelles optimales favorisant les transferts d'acyles au détriment des réactions compétitives $d^{\prime}$ hydrolyse. Les $a_{w}$ optimales pour la majorité des préparations lipasiques sont généralement comprises entre 0,25 et 0,45 , ce qui correspond le plus souvent à des teneurs en eau comprises entre 0,5 et $1 \%[19,20]$. Lorsque l'on étudie l'effet du niveau d'hydratation et de facto celui de la teneur en eau d'une lipase sur sa capacité à catalyser un transfert d'acyle, une courbe gaussienne similaire à celle présentée en figure 1 est généralement obtenue. Dans cet exemple relatif à l'aptitude des lipases du latex de Carica papaya à biocatalyser la réaction d'alcoolyse entre la trilaurine et le butanol, plusieurs essais ont été conduits avec diverses préparations enzymatiques, pré-équilibrées à différentes valeurs $d^{\prime} a_{w}$ comprises entre 0 et 1 [21]. La courbe en cloche obtenue montre clairement l'effet de l' $a_{w}$ sur l'activité catalytique des lipases pour la synthèse d'esters. Dans ce cas particulier, la formation optimale d'esters a été obtenue avec un bioca-

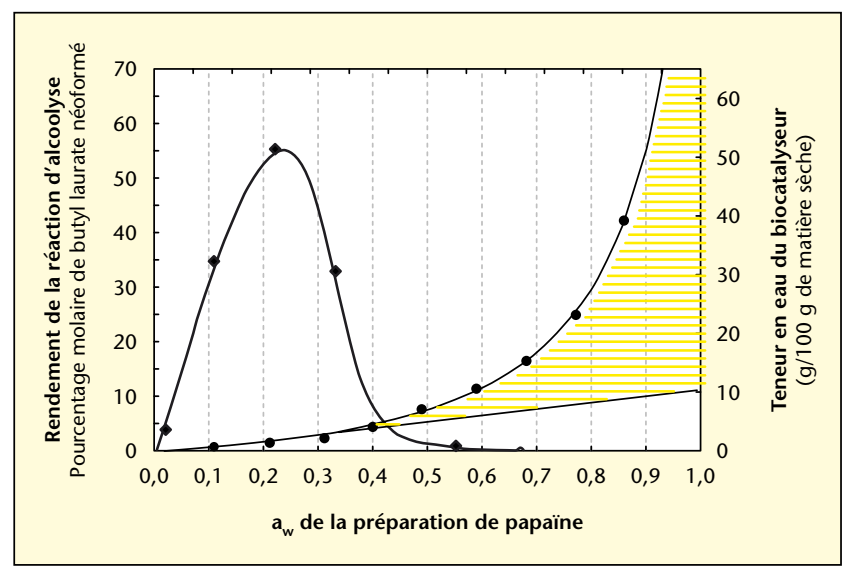

Figure 1. Influence de la variation du taux d'hydratation du biocatalyseur ( $a_{w}$ et teneur en eau) sur le rendement de la réaction d'alcoolyse de la trilaurine avec $d u$ butanol en présence de la lipase de latex brute de Carica papaya (conditions réactionnelles : $55^{\circ} \mathrm{C}, 10 \% \mathrm{~m} / \mathrm{m}$ d'enzyme brute, milieu fondu). $a_{w}$ optimale de la lipase pour la synthèse : 0,22 (correspondant à une teneur en eau du biocatalyseur de $2 \%$ ). 
talyseur prééquilibré à une valeur $\mathrm{d}^{\prime} \mathrm{a}_{w}$ de 0,22 , ce qui correspond à une teneur en eau de $2 \%$. Pour les valeurs $d^{\prime} a_{w}$ supérieures à 0,22 , une décroissance graduelle dans les rendements est observée, approchant une valeur nulle pour une estérification conduite avec une $a_{w}$ de 0,7 . Il est également primordial, lors d'une étude de l'influence de l'a $a_{w}$ sur la réaction, de tracer la courbe de sorption d'eau du biocatalyseur (figure 1) afin de définir ses différents niveaux d'hydratation. Dans l'exemple cité, il convient de signaler que l' $\mathrm{a}_{\mathrm{w}}$ optimale de la lipase est située sur la section linéaire de la courbe de sorption $\left(0,05<a_{w}<0,35\right)$, pour laquelle les molécules d'eau peuvent former des couches mono- ou multimoléculaires avec le réseau protéique. Pour des valeurs $d^{\prime} a_{w}$ supérieures à l'optimum de 0,22, une quantité excessive d'eau est adsorbée par l'intermédiaire de forces osmotiques et capillaires, ce qui favorise la réaction d'hydrolyse au détriment de la synthèse. II est particulièrement intéressant de noter qu'une valeur $d^{\prime} a_{w}$ supérieure à 0,35 correspond à la limite entre l'eau fortement liée au matériel sec et l'eau de solvatation adsorbée par les forces osmotiques et capillaires.

Bien d'autres paramètres peuvent directement influencer les cinétiques et les rendements d'estérification. Étant donné que les réactions enzymatiques sont réversibles, il est avantageux d'opérer sous des conditions réactionnelles permettant le déplacement de l'équilibre vers la synthèse. À ce sujet, diverses stratégies peuvent être adoptées. Dans un premier temps, le ratio molaire entre les deux composés initiaux à combiner peut être ajusté dans le but d'obtenir un excès de l'un des deux substrats (en général le substrat lipidique), ce qui favorise l'estérification. La nature du donneur d'acyle influence également l'efficacité de la réaction. Par exemple, I'utilisation d'acides gras libres dans une procédure d'estérification directe génère la formation d'eau qu'il est préférable d'éliminer en continu de manière à déplacer l'équilibre de la réaction vers la formation d'esters, tout en limitant I'hydrolyse inverse. Ceci est généralement rendu possible, entre autres, grâce à l'utilisation de tamis moléculaires ou en travaillant sous pression réduite. De manière alternative, d'autres donneurs d'acyles peuvent être employés, comme les esters méthyliques, éthyliques ou vinyliques. Dans ce cas, une réaction de transestérification a lieu. L'utilisation de tels esters est généralement avantageuse par rapport aux acides gras libres, puisque l'élimination des alcools formés est thermiquement plus aisée que l'élimination de l'eau. Dans le cas des esters vinyliques, la réaction est particulière, dans la mesure où ces composés sont des donneurs d'acyles irréversibles. En raison des effets tautomères, I'alcool vinylique obtenu par transestérification est transformé en acétaldéhyde, lequel est facilement retiré du milieu réactionnel du fait de son point d'ébullition très faible. Par conséquent, l'équilibre est rapidement déplacé vers la synthèse et les esters vinyliques sont donc considérés comme de très bons donneurs d'acyles pour ce type de réactions. Il a toutefois été montré que la formation de base de Schiff, par addition de l'acétaldéhyde sur les groupes aminés libres (lysine, arginine) de l'enzyme, pouvait conduire à sa dénaturation et à une perte significative d'activité [22].

Il existe également certaines stratégies au travers desquelles le composé hydrophile est chimiquement modifié lors d'une étape préliminaire. Ces modifications visent principalement à abaisser la polarité du produit, ce qui a pour conséquence d'augmenter sa solubilité dans les solvants sélectionnés et donc d'améliorer le contact entre l'enzyme et les deux substrats. La plus commune de ces modifications consiste en une alkylation préalable du composé hydrophile, entraînant la formation d'un pont éther. Les dérivés méthyliques, éthyliques, propyliques ou butylalkyliques de glucides sont également intéressants pour certaines réactions de lipophilisation biocatalysée, malgré le fait que cette stratégie nécessite une étape supplémentaire de déprotonation à la fin du procédé [23-25]. Les groupes protecteurs de type isopropylidène, benzylidène ou cyclohexylidène sont aussi fréquemment employés pour l'hydrophobation, ce qui améliore la solubilité du substrat dans les solvants sélectionnés. De nombreux exemples peuvent être trouvés dans la littérature dans lesquels les substrats hydrophiles modifiés sont acylés par un synthon lipophile et ensuite déprotégés de manière classique par des réactions chimiques impliquant une déprotection douce [26-29]. L'autre forme de modification préliminaire de substrats hydrophiles consiste en une complexation via des acides organiques boroniques tel que l'acide phényl (ou butyl) boronique [30, 31]. Ces composés sont connus pour former des complexes de boronates avec les diols et les glucides, s'accompagnant d'une amélioration de la solubilité de ces derniers dans les solvants organiques. Cependant, un effet inhibiteur de l'acide boronique a quelquefois été observé sur l'activité lipasique, notamment lorsque ce réactif est utilisé en excès [10]. Notons cependant que ces stratégies d'hydrophobations chimiques du substrat hydrophile avant la catalyse enzymatique sont très peu employées pour la lipophilisation de dérivés phénoliques. Enfin, il faut bien avoir présent à l'esprit que l'efficacité réelle de la réaction est multifactorielle. Ainsi, l'influence des conditions expérimentales telles que la température, la pression, la quantité d'enzyme ainsi que la nature du bioréacteur (batch, lit catalytique...) est très importante.

\section{Lipophilisation par catalyse lipasique de composés phénoliques}

De nombreux types de substrats peuvent être soumis à l'action des lipases dans des réactions d'estérification par les alcools ou les acides gras. Ainsi, la lipophilisation de molécules complexes comme les polyphénols et leurs dérivés pour en ajuster les propriétés physico-chimiques a été particulièrement étudiée et fait l'objet d'une abondante littérature.

\section{Lipophilisation d'acides phénoliques issus d'extraits de café vert}

En fonction de la variété de café, les acides phénoliques de Coffea arabica ou de Coffea robusta représentent de 5,5 à $12 \%$ de la matière sèche du café vert. Ces acides phénoliques sont des dérivés hydroxylés (acide caféique) ou méthoxylés (acide férulique) de l'acide cinnamique. Ils se présentent soit sous forme libre, soit sous forme d'esters ou de diesters de l'acide quinique et constituent dans ce dernier cas un groupe de composés appelés acides chlorogéniques [32] parmi lesquels l'acide 5-caféoylquinique est le constituant majoritaire (figure 2). Ces composés, partiellement solubles dans l'eau, montrent de puissantes activités antioxydantes assorties de propriétés bactéricides [33, 34]. Dans ce contexte, nous avons envisagé la possibilité de lipophiliser les acides

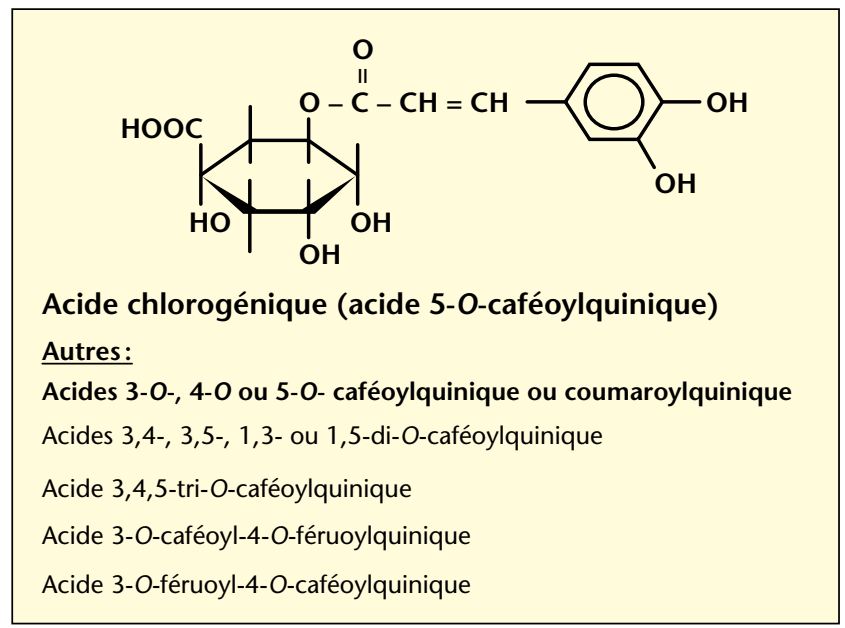

Figure 2. Structure chimique des acides chlorogéniques. 
chlorogéniques en vue d'obtenir de nouvelles biomolécules combinant des propriétés émulsifiantes, antioxydantes et antimicrobiennes [35, 36]. Dans un premier temps, nous avons étudié la capacité de la lipase de Candida antarctica B à catalyser l'estérification des acides phénoliques issus du café vert, avec des alcools gras de longueur de chaînes variables (C4 à C18) (figure 3). Typiquement, les réactions sont réalisées à $60^{\circ} \mathrm{C}$, avec une quantité d'enzyme représentant $2,5 \%$ de la masse totale des substrats, en absence de solvant. Dans un premier temps, la faisabilité de cette première réaction a été évaluée avec du butanol et les acides cinnamique, caféique, férulique et diméthoxycinnamique. Les résultats ont montré que l'estérification catalysée par des lipases avec cet alcool était possible pour les séries issues de l'acide cinnamique, à condition que le cycle aromatique ne soit pas para-hydroxylé. En effet, tandis que I'acide cinnamique et l'acide 3,4-diméthoxycinnamique ont été estérifiés avec des rendements satisfaisants, respectivement de 97 et $60 \%$, la formation d'esters n'a pas été observée dans le cas des acides caféique (acide 3,4-dihydroxycinnamique) et férulique (acide 4-hydroxy-3-méthoxycinnamique). De plus, il a été constaté qu'en présence d'une chaîne carboxylique saturée sur l'acide phénolique (acide dihydroxycaféique), la para-hydroxylation n'exerçait aucun effet. Ainsi, il semblerait que la présence simultanée d'une double liaison sur la chaîne carboxylique conjuguée avec le cycle aromatique et I'hydroxyle en para, inhibe totalement la lipase de Candida antarctica $B$. Ce phénomène semble davantage lié à un effet électronique qu'à un simple encombrement stérique, dans la mesure où l'acide 3,4-diméthoxycinnamique était convenablement estérifié, alors que les acides caféique et férulique ne l'étaient pas. L'effet de la longueur de chaîne de l'alcool sur le taux d'estérification a ensuite été évalué avec l'éthanol, le butanol, l'octanol, le dodécanol et l'alcool oléique. Là encore, il a été montré que l'estérification de l'acide caféique n'était pas possible, et ce, quel que soit l'alcool employé. Pour tenter de comprendre ces divers phénomènes et pour expliciter les différences significatives en termes de réactivité entre tous ces acides phénoliques, la réaction par voie enzymatique a été effectuée sur deux familles de composés, à savoir les isomères des acides hydroxycinnamiques et benzoïques et les isomères de l'acide dihydroxybenzoïque, en appliquant la même procédure utilisant l'octanol comme alcool. II a été constaté que l'estérification des isomères de l'acide hydroxycinnamique était possible, mais lente. Le meilleur rendement a été obtenu avec les isomères en méta, tandis que ceux en ortho et para se comportaient de manière similaire. Ce résultat a été partiellement confirmé par I'estérification des isomères de l'acide hydroxybenzoïque, avec toutefois de faibles rendements. Par contre, les réactions impliquant les acides dihydroxybenzoïques n'ont conduit qu'à l'obtention d'esters à l'état de traces, et cela même, après plusieurs jours de réaction. À partir de ces résultats, on peut également conclure que la présence de deux fonctions hydroxyles sur le cycle aromatique inhibe l'estérification biocatalysée de la fonction acide, si cette dernière est conjuguée avec le cycle, soit par une liaison sigma directe sur le cycle (acide benzoïque), soit par l'intermédiaire d'une double liaison sigma-pi (acide cinnamique).

In fine, ce constat a été extrapolé à la synthèse enzymatique d'esters gras de l'acide 5-caféoylquinique. La réaction d'estérification a été étudiée

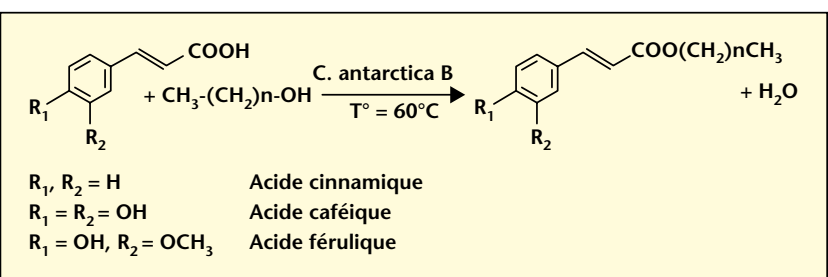

Figure 3. Estérification par voie enzymatique d'acides phénoliques de café vert avec différents alcools gras. avec différents alcools (C8, C12, C16 et C18: 1) en utilisant la lipase de Candida antarctica B comme biocatalyseur (1,5\% p/p) (figure 4). Deux types de milieu réactionnel ont été utilisés, à savoir avec le 2-méthyl-2butanol ou sans solvant. Dans ce dernier cas, l'estérification par des alcools gras a été possible et il s'est avéré que la conversion était dépendante de la longueur de la chaîne carbonée. Sa valeur était d'environ $60 \%$ pour les alcools en C8, et $40 \%$ pour les alcools de $\mathrm{C} 12$ à C18 après 30 jours de réaction. Notons qu'à l'instar des acides phénoliques de la série issue de l'acide cinnamique, l'estérification est très lente. En présence de 2-méthyl-2-butanol, les réactions ont été plus rapides et les conversions ont été systématiquement plus élevées que celles obtenues en milieu fondu, s'échelonnant de 55 à $75 \%$ en fonction de la longueur de chaîne de l'alcool. Dans tous les cas, les cinétiques d'estérification se sont rapidement accélérées après 4 jours. Cette accélération typique pourrait s'expliquer par les propriétés émulsifiantes des esters néoformés dont la présence, même en faibles quantités, est suffisante y-pour améliorer la solubilité des réactifs.

\section{Lipophilisation d'autres dérivés phénoliques}

L'estérification enzymatique d'acides phénoliques a été étudiée par différents auteurs. Tout d'abord, Buisman et al. [37] ont évalué deux stratégies d'estérification par des alcools gras. L'une s'inscrivait dans le prolongement du travail décrit par Guyot et al. [35] concernant l'estérification d'acides cinnamiques, tandis que l'autre étude traitait des composés aromatiques comprenant des fonctions alcools primaires qui peuvent être estérifiées par des acides aliphatiques. En ce qui concerne les dérivés $d^{\prime}$ acides cinnamiques et benzoïques, I'estérification a été testée avec le butanol, en présence de différentes lipases (Candida antarctica B, Humicola lanuginosa, Candida rugosa, Pseudomonas sp et Geotrichum candidum). Pour toutes les enzymes testées, excepté celle issue de Candida antarctica $B$, des rendements particulièrement faibles et n'excédant pas $3 \%$ ont été observés. Par conséquent, la lipase de Candida antarctica $B$ a été choisie pour les expériences suivantes et l'effet de la nature du solvant organique utilisé dans la réaction a été évalué. Dans les solvants apolaires tels que l'heptane ou le cyclohexane, des conversions supérieures à $85 \%$ ont été observées en moins de 5 jours. En revanche, les cinétiques réactionnelles ont été extrêmement lentes avec des solvants plus polaires, comme l'éther diéthylique, le tert-butylméthyl éther et le butanol. Les résultats fournis par Guyot et al. [35] pour l'estérification de l'acide benzoïque et de ses dérivés ont ainsi été confirmés dans cette étude. Les auteurs ne constatent pratiquement aucune conversion pour les différents substituants hydroxy et méthoxy de l'acide benzoïque. D'ailleurs, l'effet inhibiteur de la conjugaison des substituants donneurs d'électrons au groupe carboxylique a de nouveau été mis en évidence. II est avancé que de tels effets de donneurs d'électrons peuvent induire une désactivation intrinsèque du centre carbone électrophile du groupe carboxylique pour l'attaque nucléophile de l'alcool.

La seconde stratégie examinée par ces auteurs consiste à lipophiliser des phénols comportant des fonctions hydroxyles primaires sur le cycle aromatique. La lipase de Candida antarctica B a été employée pour l'estérification de I'hydroxytyrosol et de I'alcool 3,5-di-tert-butyl-4hydroxybenzylique (figure 5). L'estérification du premier composé a été

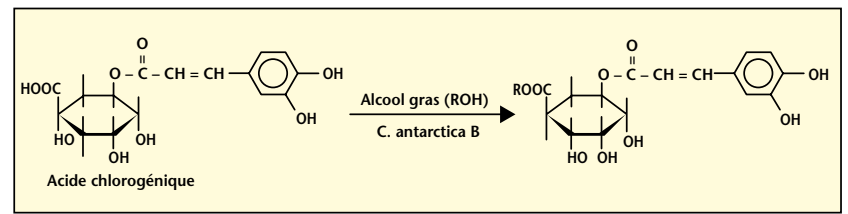

Figure 4. Synthèse d'esters gras d'acides chlorogéniques par catalyse enzymatique. Exemple de l'acide 5-caféoylquinique. 


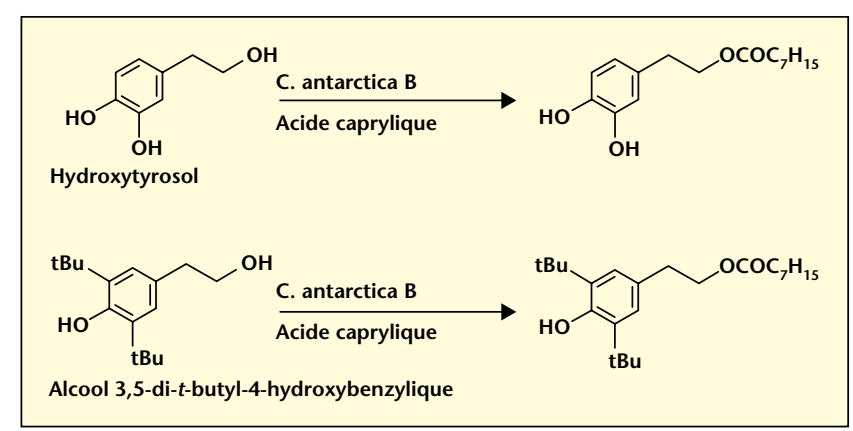

Figure 5. Estérification par catalyse lipasique d'hydroxytyrosol et d'alcool 3,5-di-tertbutyl-4-hydroxybenzylique.

opérée avec de l'acide octanoïque dans différents solvants. Dans l'éther diéthylique, une conversion d'environ $85 \%$ a été obtenue après 15 jours de réaction à $35^{\circ} \mathrm{C}$. Dans les solvants plus polaires tels que le chloroforme, le dichlorométhane ou le tétrahydrofurane, des conversions proches de $20 \%$ sont constatées. Finalement, avec l'heptane et l'hexane, la formation d'esters est de l'ordre de 70 à $80 \%$. L'estérification complète de l'alcool 3,5-di-tert-butyl-4-hydroxybenzylique, a été obtenue en moins de 2,5 heures à $81^{\circ} \mathrm{C}$ dans du cyclohexane.

Priaya et al. [38] ont évalué les potentialités de la lipase de Pseudomonas cepacia à catalyser la réaction de transestérification de dérivés cinnamates avec différents alcools (butanol, alcool benzylique, octanol et phényléthanol). Seul le butanol a permis de former le produit transestérifié. Pour optimiser la réaction, l'effet de la nature du solvant, dépendant de sa valeur de $\log P$, a été étudié. II a été constaté que les solvants présentant une valeur élevée de $\log P$, comme le cyclohexane, I'heptane ou I'hexane, fournissent les meilleurs résultats. Dans tous ces cas de figure, une conversion supérieure à $90 \%$ a été obtenue en 48 heures, l'éther diisopropylique et le toluène étant les solvants les plus appropriés pour ce type de réaction.

Finalement, Stamatis et al. [39] ont évalué l'aptitude des lipases de Candida antarctica $\mathrm{B}$ et de Rhizomucor miehei à catalyser la synthèse de dérivés lipophiles d'antioxydants naturels issus de l'acide cinnamique. En premier lieu, l'estérification de l'acide cinnamique avec l'octanol a été choisie comme réaction modèle, avec les deux lipases immobilisées, dans différents solvants non toxiques tels que l'acétone, le 2-méthyl-2propanol et le 2-méthyl-2-butanol. II a été montré que le rendement de réaction était dépendant du solvant et de la lipase utilisée. Avec la lipase de Candida antarctica B, l'octyl cinnamate a été formé avec des rendements supérieurs à $80 \%$. II est apparu également que des rendements plus élevés étaient obtenus en utilisant l'octanol en large excès. De telles conditions réactionnelles rendent possible la solubilisation partielle de l'acide cinnamique dans l'alcool. L'estérification de l'acide cinnamique en absence de solvant a ensuite été testée en employant des alcools primaires de longueurs de chaîne différentes (C4 à C10). II a été observé que l'activité catalytique de la lipase est influencée par la longueur de chaîne de l'alcool. Les effets de la structure des acides phénoliques ont également été étudiés. Différents dérivés hydroxylés ou méthoxylés de l'acide cinnamique, mais également des acides hydroxybenzoïques et phénylacétiques, ont été estérifiés avec de l'octanol. Les auteurs montrent que les dérivés des acides cinnamiques et benzoïques dont I'hydroxyle est substitué, et plus spécialement les isomères ortho et para, inhibent l'action catalytique des deux lipases. Lorsque la chaîne carboxylique est saturée (acide para-hydroxyphénylacétique), I'hydroxylation en para n'exerce aucun effet sur la lipase. Ainsi, ce travail confirme de nouveau, ce qui avait été précédemment observé par Guyot et al. puis par Buisman et al. D'autres équipes ont également travaillé sur la synthèse biocatalysés d'esters féruliques. Par exemple, Compton et al. [40]<smiles>[R5]c1cc(C2Oc3cc(O)cc(O)c3CC2[R])c([R])c([R])c1[R5]</smiles>

Flavonoïdes<smiles>[R]c1cc(-c2oc3cc(O)cc(O)c3c(=O)c2O)c([R])c([R])c1[R]</smiles>

Flavonols<smiles></smiles>

Anthocyanidines
Figure 6. Structure chimique de quelques flavonoïdes.

ont utilisé la lipase de Candida antarctica B pour la synthèse d'octyl ferulate soit par estérification directe en milieu 2-méthyl-2- propanol soit par alcoolyse de l'éthyl ferulate en milieu fondu dans l'octanol. Ces auteurs ont étendu le procédé au greffage d'acide ferulique sur des triacylglycérols avec un rendement de $77 \%$ concernant l'obtention de féruloyl mono ou dioléine. De manière identique, tout récemment, une équipe canadienne a également travaillé sur le greffage direct d'acides phénoliques sur un motif triglycéridique. Ainsi I'acide dihydrocaféique a été transestérifié tout d'abord sur la trilinoléine ou trilinolénine [41] puis, sur de l'huile de lin [42] avec différents ratios de mono- et diacylglycérols phénoliques obtenus suivant les paramètres réactionnels choisis (rapport des substrats, milieu réactionnel : hexane/butanone).

\section{Lipophilisation par voie enzymatique de flavonoïdes glycosylés}

Les flavonoïdes, molécules attractives en raison de leurs fortes propriétés antioxydantes, sont présents dans différents fruits, légumes, épices ou herbes [43] (figure 6). II semble toutefois que la nature hydrophile de ces antioxydants réduise leur efficacité à protéger les huiles et les graisses contre les processus oxydatifs. Certains exemples traitant de l'utilisation des lipases pour la lipophilisation de ces molécules ont été publiés récemment. Ainsi, citons Kontogianni et al. [44, 45] qui ont réalisé l'acylation enzymatique de la naringine (naringénine-2-0rhamnosylglucose) (figure 7) et de la rutine (quercétine-3-0rhamnosylglucose). Pour estérifier la naringine avec des acides gras à chaînes moyennes (C8, C10, C12), la lipase de Candida antarctica B a été utilisée et différents paramètres réactionnels ont été évalués de manière à travailler avec les milieux les moins toxiques (acétone, tétrahydrofurane et tert-butanol). Lorsque l'acide octanoïque est utilisé en tant que donneur d'acyles, les taux de conversion les plus élevés ont été observés dans

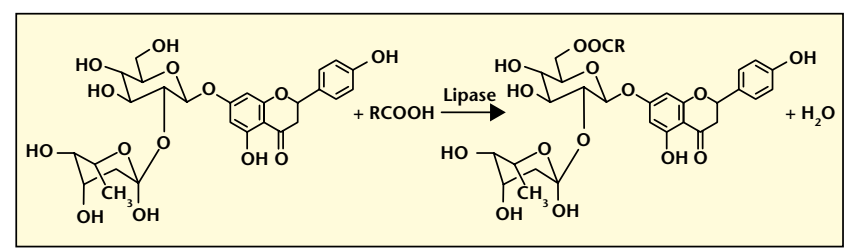

Figure 7. Acylation régiosélective de la naringine. 
I'acétone, tandis que pour les acides décanoïque et dodécanoïque, le tert-butanol s'est avéré être le meilleur solvant. En revanche, le tétrahydrofurane n'a été satisfaisant pour aucun des substrats. Qui plus est, la caractérisation des produits par différentes techniques analytiques démontre clairement que l'acylation s'est effectuée de manière hautement régiosélective, générant un seul produit correspondant à l'estérification de la seule fonction hydroxyle primaire de la naringine. L'effet de $l^{\prime} a_{w}$ du milieu réactionnel a également été évalué en utilisant du tertbutanol comme solvant. Les conversions les plus élevées ont été obtenues pour de très faibles valeurs $d^{\prime} a_{w}$, proches de 0,11 ou moins. De même, une autre étude traite de l'estérification de ces flavonoïdes glycosylés via des acides gras à plus longues chaînes tel que l'acide palmitique [46]. Dans ce cas, les réactions ont été conduites dans du 2-méthyl-2-butanol et les rendements de formation de la naringine 6-0-palmitate sont de $43 \%$ environ après 55 heures de réaction.

\section{Propriétés antioxydantes des composés phénoliques lipophilisés}

\section{Interface et paradoxe polaire}

Le paramètre essentiel de l'activité antioxydante en milieu apolaire concerne la localisation de l'antioxydant au sein du système. En effet, plus I'antioxydant est proche de l'endroit où a lieu l'oxydation, plus il est efficace. Ceci est bien illustré par le paradoxe polaire de Porter, selon lequel les antioxydants polaires sont plus souvent actifs dans les solutions lipidiques homogènes que les antioxydants apolaires, tandis que ces derniers sont plus efficaces dans les milieux émulsionnés que leurs homologues polaires [47-52]. Ce paradoxe est fondé sur les propriétés interfaciales des antioxydants, sur leur partition dans les milieux multiphasiques et sur le fait crucial que l'oxydation des lipides est initiée aux interfaces du système. Ceci peut se comprendre en simplifiant l'oxydation de la manière suivante : les agents initiant l'oxydation lipidique sont polaires, alors que les cibles de l'oxydation (les lipides insaturés) sont par nature lipophiles et donc préférentiellement orientés vers la phase apolaire. L'interface qu'elle soit de type huile/air ou eau/huile constitue donc le lieu privilégié où se rencontrent ces deux « acteurs » de l'oxydation. Ainsi, dans une solution lipidique homogène, l'oxydation a lieu à l'interface air/huile où les antioxydants hydrophiles sont concentrés, tandis qu'en milieu émulsionné, elle à lieu à l'interface huile/eau, où sont localisés les antioxydants lipophiles. Ce comportement paradoxal a été confirmé, entre autres, par le fait que l'acide ascorbique, les acides carnosiques et rosmariniques issues du romarin ainsi que le Trolox (un équivalent hydrophile de l'a-tocophérol) sont de meilleurs antioxydants en milieu lipidique homogène que leurs homologues lipophiles ( $\alpha$-tocophérol, ascorbyl palmitate et carnosol). II faut également avoir présent à l'esprit que la capacité d'une molécule antioxydante à protéger un substrat lipidique peut varier de manière significative selon que ce substrat oxydable soit dans un environnement triglycéridique, sous forme d'ester méthylique, d'acide gras libre ou encore incorporés au sein de différentes particules biologiques, telles que les lipoprotéines de basse densité ou les structures membranaires de type liposomes ou microsomes de foie. En outre, au sein de la phase aqueuse, l'efficacité des antioxydants hydrophiles peut être contrebalancée par leur activité prooxydante qui résulte de la réduction de certains métaux oxydant en leur forme plus active. Enfin, en préalable à toute procédure de lipophilisation, il faut bien prendre en considération le fait que la capacité antioxydante d'un composé phénolique s'exerce par l'intermédiaire des fonctions alcools directement liées au cycle aromatique. Ainsi, en théorie, la réaction d'hydrophobation ne doit pas affecter les groupements hydroxyles impliqués dans ce pouvoir antioxydant.

\section{Capacité antioxydante des molécules lipophilisées}

Les composés phénoliques présentent un fort potentiel antiradicalaire, d'une part en raison de leur capacité à céder un atome d'hydrogène (proton et électron) à un radical libre et d'autre part, grâce à la bonne stabilité du radical aryloxyl ainsi formé. Il existe un nombre considérable d'études traitant des effets antioxydants de composés phénoliques purifiés ou de différents extraits végétaux riches en ces substances. En revanche, malgré de considérables efforts [53], la connaissance du pouvoir antioxydant des composés phénoliques lipophilisés demeure parcellaire et leur valorisation limitée.

Concernant les acides phénoliques simples, Sabally et al. [41, 42] ont utilisé la méthode DPPH (diphénylpicrylhydrazyl) pour évaluer le pouvoir antiradicalaire d'acide dihydrocaféique estérifié par des triacylglycérols de type trilinoléine, trilinolénine ou triacylglycerols de lin. S'il apparaît que ces molécules expriment globalement une bonne activité antiradicalaire, il est toutefois à noter que le greffage de l'acide dihydrocaféique sur une matrice triglycéridique ne permet pas d'améliorer l'activité antiradicalaire en rapport à la molécule de départ. Les auteurs attribuent cette perte d'activité à un changement conformationnel de l'acide. Par ailleurs, cette même équipe a constaté que l'estérification directe d'alcools gras linoléique ou linolénique sur l'acide dihydrocaféique induit également une perte de l'activité antiradicalaire vis-à-vis du radical libre $\mathrm{DPPH}^{\bullet}[54,55]$. Cependant, le fait que les molécules lipophilisées par des alcools gras présentent une meilleure activité antiradicalaire que celles obtenues par greffage de triacylglycérols, permet aux auteurs de conclure que la nature de la chaîne aliphatique greffés est de prime importance quant à l'activité antiradicalaire. En effet, il semblerait qu'un groupement lipophile de fort encombrement stérique limite la rotation de la chaîne latérale de l'acide phénolique lipophilisé, ce qui s'accompagnerait d'une baisse de l'activité antiradicalaire. Cependant, on peut également suspecter que la méthode DPPH telle que mise en œuvre dans ces travaux ne soit pas adaptée à la mesure d'activité antiradicalaire pour les dérivés triglycéridiques, et ce, en raison de la nature du milieu organique choisi (éthanol). Amselmi et al. [56] ont quant à eux, mené une étude plus complète de l'activité antioxydante de molécules lipophilisées de type alkyl ferulates, en solutions aqueuse (ORAC, oxygen radical absorbance capacity) et alcoolique (DPPH) ainsi qu'en suspensions de microsomes où l'activité antioxydante a été mesurée par la méthode TBARS (substances réactives à l'acide thiobarbiturique) (tableau 2, figure 8). Concernant la synthèse, des chaînes grasses de structure linéaire ou ramifiée et de longueurs s'échelonnant de six à dix huit atomes de carbones ont été testés. Les auteurs constatent que la lipophilisation ne modifie pas significativement le pouvoir antioxydant en milieu polaire (DPPH et ORAC) et concluent, par conséquent, que la nature de la chaîne latérale n'exerce aucune influence vis-à-vis des interactions structurales liées à l'activité antiradicalaire. A contrario, une forte amélioration du pouvoir antioxydant des molécules lipophilisées en rapport aux molécules initiales a été observée en suspension microsomiale. De manière surprenante, les auteurs n'attribuent pas ce phénomène à la différence de polarité des molécules - mesurées expérimentalement par le log $\mathrm{P}$ - mais plutôt à une interaction de type molécule lipophilisée/membrane phospholipidique qui dépend de la nature de la chaîne aliphatique greffée. Par conséquent, les auteurs ont fait appel à la RMN et aux outils de modélisation pour caractériser ces interactions. Les résultats montrent d'une part, que la liaison avec la membrane phospholipidique est plus stable quand la chaîne latérale de la molécule présente une flexibilité conformationelle élevée et d'autre part, lorsque la stabilité du champ conformationnel est maximale (énergie de liaison minimale), ce qui a bien été démontré pour la chaîne en $\mathrm{C} 12$ (A6, figure 8 ).

L'activité antioxydante de l'acide 3,4-dihydroxymandélique lipophilisé par des amines aliphatiques ( 6 à 8 carbones, de type linéaire ou ramifiée) a récemment été évaluée [57] par différentes stratégies de mesure, parmi 
Tableau 2. Capacités antioxydantes de dérivés lipophiles d'acide férulique.

\begin{tabular}{|lccc|}
\hline Composé & DPPH $^{\mathbf{a}}$ & ORAC $^{\mathbf{b}}$ & TBARS $^{\mathbf{c}}$ \\
\hline A & $21,98 \pm 2,13$ & $31,10 \pm 2,15$ & 250,11 \\
A1 & $22,66 \pm 1,57$ & $28,60 \pm 1,62$ & 27,54 \\
$A 2$ & $22,94 \pm 1,38$ & $29,99 \pm 2,19$ & 12,40 \\
A3 & $25,53 \pm 1,68$ & $28,85 \pm 1,80$ & 33,16 \\
A4 & $24,64 \pm 1,89$ & $28,46 \pm 2,22$ & 19,74 \\
A5 & $21,33 \pm 2,82$ & $28,31 \pm 1,88$ & 31,61 \\
A6 & $26,62 \pm 2,57$ & $26,99 \pm 1,95$ & 11,03 \\
A7 & $25,60 \pm 1,25$ & $28,57 \pm 3,13$ & 18,64 \\
A8 & $24,93 \pm 1,63$ & $28,80 \pm 2,73$ & 30,45 \\
A9 & $25,47 \pm 1,35$ & $28,46 \pm 0,68$ & 40,79 \\
A10 & $24,97 \pm 1,95$ & $30,67 \pm 1,14$ & 49,78 \\
\hline
\end{tabular}

a Exprimé en $\mathrm{IC}_{50}(\mu \mathrm{M})$; solution méthanolique de DPPH $(50 \mu \mathrm{M})$; antioxydants $(5-50 \mu \mathrm{M})$.

${ }^{\mathrm{b}}$ Exprimé en équivalents Trolox $(\mu \mathrm{M})$; solution aqueuse (tampon phosphate) de R-phycoérythrine (16,7 nM) ; initiateur 2,2'-azobis(2-amidinopropane) dihydrochloride $(3 \mathrm{mg} / \mathrm{ml})$; antioxydants $(5-50 \mu \mathrm{M})$.

c Exprimé en $\mathrm{IC}_{50}(\mu \mathrm{M})$; suspension aqueuse (tampon phosphate) de microsomes de foie de rat (concentration inconnue); initiateur $\mathrm{Fe}^{+3} /$ acide ascorbique (1,5 $\mu \mathrm{M})$; antioxydant $(5-50 \mu \mathrm{M})$.

lesquelles nous aborderons plus particulièrement (i) le test DPPH, (ii) le test d'activité de piégeur d'anion superoxyde et (iii) la méthode de co-oxydation de l'acide linoléique et du $\beta$-carotène (tableau 3, figure 9). En premier lieu, il a été observé que l'acide 3,4-dihydromandélique, qu'il soit lipophilisé ou non, exerçait une plus grande activité antioxydante que l'acide ascorbique, l' $\alpha$-tocophérol, le BHA et le BHT, qui sont des antioxydants de référence. Les résultats concernant l'influence de la

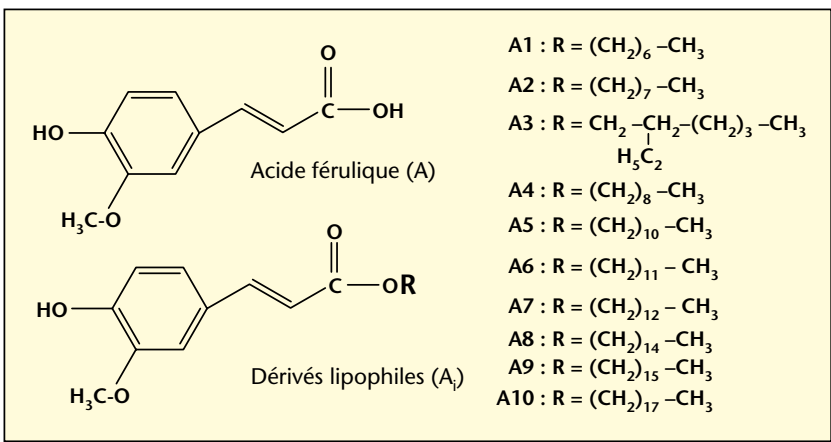

Figure 8. Structure chimique de l'acide férulique et de ces dérivés lipophiles.

Tableau 3. Capacités antioxydantes de dérivés lipophiles d'acide 3,4-dihydroxymandélique.

\begin{tabular}{|lccc|}
\hline Composé & DPPH $^{\mathrm{a}}$ & $\begin{array}{c}\text { Anion } \\
\text { superoxyde }^{b}\end{array}$ & $\boldsymbol{\beta}_{\text {-carotène }}{ }^{\mathrm{c}}$ \\
\hline$B$ & $0,062 \pm 0,005$ & 130 & $0,77 \pm 0,04$ \\
$B 1$ & $0,120 \pm 0,004$ & $220 \pm 70$ & $0,38 \pm 0,12$ \\
$B 2$ & $0,112 \pm 0,004$ & $64 \pm 34$ & $n \cdot d$. \\
$B 3$ & $0,090 \pm 0,009$ & $n \cdot d$. & $0,19 \pm 0,08$ \\
$B 4$ & $0,116 \pm 0,001$ & $580 \pm 130$ & $0,27 \pm 0,03$ \\
\hline
\end{tabular}

n.d., non déterminé.

a Exprimé en $\mathrm{EC}_{50}(\mathrm{~mol} / \mathrm{mol})$.

${ }^{\mathrm{b}}$ Exprimé en $\mathrm{IC}_{50}(\mathrm{nM})$.

c Exprimé en $\mathrm{IC}_{50}(\mathrm{mM})$.

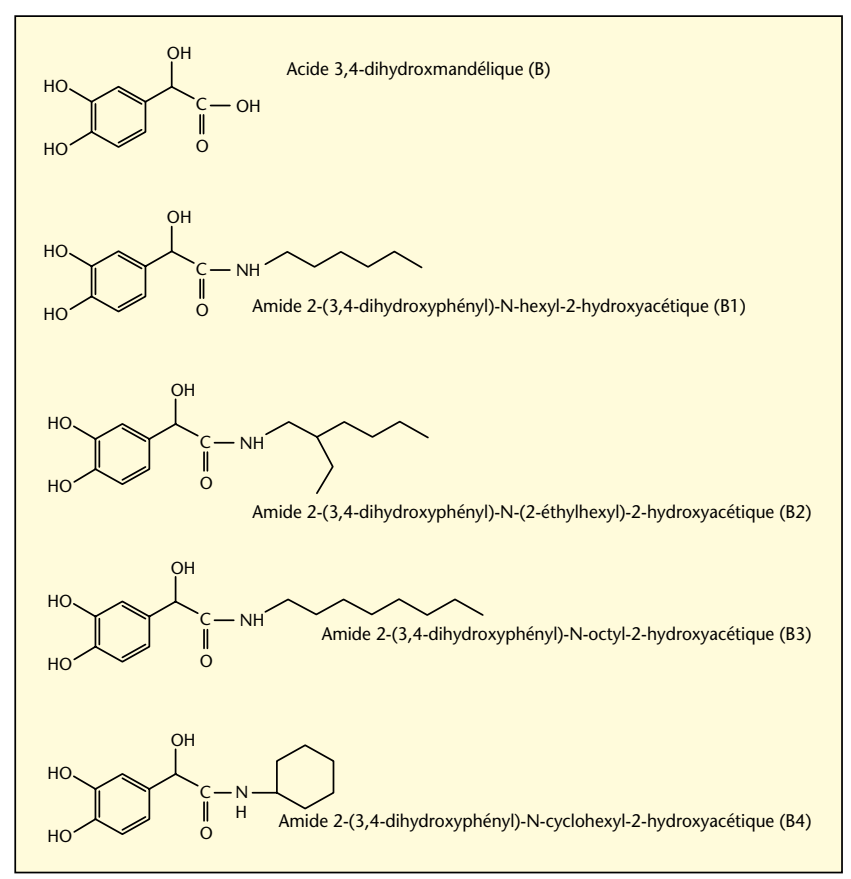

Figure 9. Structure chimique de l'acide 3,4-dihydroxymandélique et de ces dérivés lipophiles.

lipophilisation sur l'activité antioxydante sont en revanche plus mitigés. Pour le test DPPH, toutes les molécules lipophilisées ont vu leur activité antiradicalaire diminuée. À l'opposé, une amélioration de la capacité de ces molécules à piéger l'anion superoxyde a été observé seulement lorsqu'une chaîne ramifiée a été greffée. En système émulsionné (acide linoléique/ $\beta$-carotène), il a finalement été constaté que plus la chaîne grasse était longue, plus le pouvoir antioxydant de la molécule hydrophobée augmentait par rapport à l'acide phénolique initial. Ce résultat montre clairement que les antioxydants lipophilisés protègent mieux le substrat lipidique que l'acide 3,4-dihydroxymandélique, puisqu'ils sont directement localisés sur le « site d'oxydation » (interface et phase lipidique). Enfin, citons une étude très récente visant à déterminer les effets de la lipophilisation de I'hydroxytyrosol par des chaînes aliphatiques de type acétate, palmitate, oléate et linoléate [58] (tableau 4, figure 10). Les résultats montrent que la lipophilisation induit une augmentation de l'activité antioxydante vis-à-vis des lipides en système biologique (tissus de cerveaux de rats).

Concernant les flavonoïdes, un certain nombre de travaux récents, permettent de mieux comprendre l'influence de la lipophilisation sur le comportement antioxydatif. À titre d'exemple nous citerons simplement, Mellou et al. [59] qui ont acylé deux flavonoïdes glycosylés par des chaînes lauriques en mesurant l'effet de la lipophilisation sur l'oxydation

Tableau 4. Capacités antioxydantes de dérivés lipophiles d'hydroxytyrosol.

\begin{tabular}{|lc|}
\hline Composé & TBARS $^{\mathbf{a}}$ \\
\hline Témoin & 100 \\
C & 52 \\
C1 & 60 \\
C2 & 46 \\
C3 & 48 \\
C4 & 30 \\
\hline
\end{tabular}

${ }^{a}$ Cénération de malondialdéhyde (en \%) en présence (B, B1-B4) ou en absence (Témoin) de 5 mM d'antioxydant. 


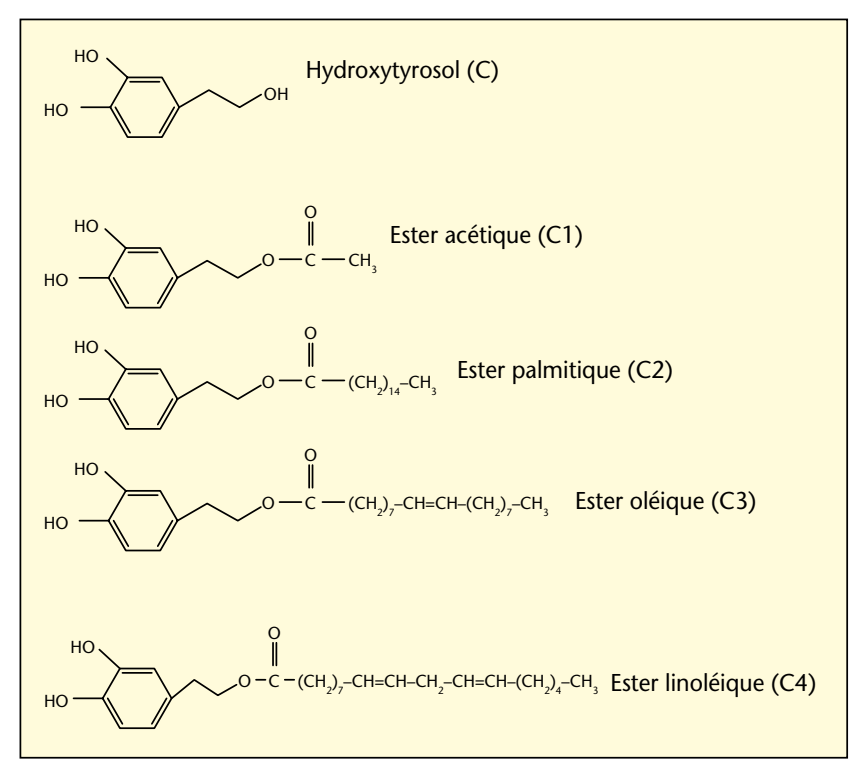

Figure 10. Structure chimique de I'hydroxytyrosol et de ses dérivés lipophiles.

du LDL. II est apparu que les molécules hydrophobées protégeaient mieux les lipoprotéines que leurs homologues non modifiés. Les auteurs attribuent principalement cet effet à une diminution de la polarité de la molécule induite par l'acylation, ce qui aurait pour effet de mieux localiser l'antioxydant au sein de la suspension. Néanmoins, il n'est pas exclu que l'amélioration des propriétés antioxydantes soit relayée de manière complémentaire par d'autres mécanismes.

\section{Conclusions et perspectives}

Les lipases sont des enzymes qui peuvent être employées pour différentes sortes de réactions biocatalysées. Elles peuvent bien souvent être considérées comme plus avantageuses que les catalyseurs chimiques classiques, dans la mesure où leur utilisation permet d'opérer de manière régiosélective dans des conditions réactionnelles douces. II semble en particulier qu'elles soient très adaptées pour la synthèse de molécules impliquant le greffage d'un synthon lipophile à une molécule hydrophile. Dans de telles réactions, différents paramètres et stratégies peuvent être adoptés, dans le but d'améliorer les cinétiques et les rendements réactionnels. Récemment, de nouvelles applications des lipases ont été décrites pour modifier des composés naturels tels que les acides phénoliques et les flavonoïdes. Ces réactions biocatalysées ont pour objectif principal de moduler la balance hydrophile/lipophile de molécules initialement hydrophiles pour conférer aux nouveaux produits des propriétés multifonctionnelles combinant par exemple des activités antioxydantes et émulsifiantes. Se pose alors la question de l'efficacité antioxydante réelle des composés lipophilisés et des outils nécessaires à leur évaluation. Or, dans ce domaine, la diversité des molécules à analyser a conduit à I'utilisation de nombreux tests d'activité antioxydante, aucun d'entre eux ne présentant malheureusement un caractère universel. Cependant, malgré la disparité des résultats obtenus, il apparaît que les molécules lipophilisées sont plus actives en émulsions et en suspension lipidique que les molécules initiales. Bien évidemment, de nombreux travaux devront encore être entrepris pour espérer une application de ces réactions biocatalysées à l'échelle industrielle ainsi qu'une valorisation des molécules à des fins nutritionnelles et thérapeutiques. À long terme, les rendements réactionnels et la performance des enzymes devront notamment être améliorés. Néanmoins, il apparaît probable qu'à l'avenir la lipophilisation biocatalysée de composés phénoliques est indubitable- ment amenée à progresser d'autant qu'elle permet l'obtention de biomolécules hautement spécifiques, à forte valeur ajoutée et très souvent multifonctionnelles.

Remerciements. Luis Javier López-Giraldo bénéficie du soutien du programme Alßan, Programme de Bourses de Haut Niveau de I'Union Européenne pour l'Amérique Latine, bourse NE05D055786CO.

\section{RÉFÉRENCES}

1. KASLAUSKAS AL, BORNSCHEUER UT. Biotransformations with Lipases. In : Rehm HJ, Reed G, eds. Biotechnology. Second edition vol 8A. Weinheim : Wiley-VCH, 1998 : 37-191.

2. MACRAE AR. Lipase-catalyzed interesterification of oils and fats. / Am Oil Chem Soc $1983 ; 60: 291-4$.

3. FOGLIA TA, VILLENEUVE P. Carica papaya latex-catalyzed synthesis of structured triacylglycerols. J Am Oil Chem Soc 1997 ; 74 : 1447-50.

4. QUINLAN P, MOORE $S$. Modification of triglycerides by lipases: process technology and its applications to the production of nutritionally improved fats. Inform $1993 ; 4$ : 580-5.

5. XU X. Modification of Oils and Fats by Lipase-catalyzed Interesterification. Aspects of process engineering. In : Bornscheuer UT, ed. Enzymes in Lipid Modification. Weinheim : Wiley-VCH, $2000: 190-215$.

6. GANDHI NN. Applications of Lipases. J Am Oil Chem Soc 1997 ; 74 : 621-34.

7. YAHYA ARM, ANDERSON WA, MOO-YOUNG M. Ester synthesis in lipasecatalyzed reactions. Enz Microb Technol $1998 ; 23: 438-50$.

8. BORNSCHEUER UT. Lipase-catalyzed synthesis of monoacylglycerols. Enz Microb Technol $1995 ; 17: 578-86$.

9. BALCAO VM, MALCATA FX. Bioreactors with immobilized lipases : state of the art. Enz Microb Technol 1996 ; 18 : 392-416.

10. LANG S, SYLDATK C, RAU U. Enzymatic synthesis and modification of glycolipids. In : Bornscheuer UT, ed. Enzymes in Lipid Modification. Weinheim : Wiley-VCH, $2000: 361-93$.

11. BAGI S, SIMON K. Comparison of esterification and transesterification of fructose by porcine pancreatic lipase immobilized on different supports. Biotechnol Tech $1999 ; 13$ : 309-12.

12. LJUNGER G, ADLERCREUTZ P, MATTIASSON B. Lipase catalyzed acylation of glucose. Biotechnol Lett $1994 ; 16: 1167-72$.

13. KU MA, HANG YD. Enzymatic synthesis of esters in organic medium with lipase from Byssochlamys fulva. Biotechnol Lett 1995 ; 17 : 1081-4.

14. DEGN P, PEDERSEN LH, DUUS J, ZIMMERMANN W. Lipase-catalyzed synthesis of glucose fatty esters in tert-butanol. Biotechnol Lett 1999; 21 : 275-80.

15. KHALED N, MONTET D, PINA M, GRAILLE I. Fructose oleate synthesis in a fixed catalyst bed reactor. Biotechnol Lett $1991 ; 13$ : 167-72.

16. DUCRET A, GIROUX A, TRANI M, LORTIE R. Characterization of enzymatically prepared biosurfactants. I Am Oil Chem Soc 1996; 73 : 109-13.

17. COULOND, GIRARDINM, GHOUL M. Enzymatic synthesis of fructose monoleate in a reduced pressure pilot scale reactor using various acyl donors. Process Biochem 1999 ; 34 : 913-8.

18. YANY, BORNSCHEUER UT, CAO L, SCHMID RD. Lipase-catalyzed solid phase synthesis of sugar fatty acid esters, removal of byproducts by azeotropic distillation. Enz Microb Tech 1999 ; 25 : 725-8.

19. VILLENEUVE P, MUDERHWA JM, HAAS MJ, GRAILLE J. Customizing lipases for biocatalysis : a survey of chemical, physical and molecular biological approaches. J Molec Cat. B Enz 2000 ; 9 : 113-48. 
20. MUDERHWA JM, PINA M, GRAILLE J. Aptitude à la transestérification de quelques lipases régiosélectives 1-3. Oléagineux $1988 ; 43$ : 427-32.

21. CARO Y, PINA M, TURON F, et al. Plant lipases : biocatalyst aqueous environment in relation to optimal catalytic activity in lipase-catalyzed synthesis reactions. Biotechnol Bioeng $2002 ; 77: 693-703$.

22. WEBER HK, STECHER H, FABER K. Sensitivity of microbial lipases to acetaldehyde formed by acyl-transfer reactions from vinyl esters. Biotechnol Lett $1995 ; 17: 803-8$.

23. BJÖRKLING F, GODTFREDSEN SE, KIRK O. A highly selective enzymecatalyzed esterification of simple glucosides. I Chem Soc Chem Commun 1989 : 934-5.

24. BISHT KS, GROSS RA, KAPLAN DL. Enzyme mediated regioselective acylations of sophorolipids. J Org Chem $1998 ; 64$ : 780-9.

25. BOUSQUET MP, WILLEMOT RM, MONSAN P, BOURES E. Enzymatic synthesis of unsaturated fatty acid glucoside esters for dermo-cosmetic applications. Biotechnol Bioeng $1999 ; 63$ : 730-6.

26. SARNEY DB, KAPELLER H, FREGAPANE G, VULFSON EN. Chemo-enzymatic synthesis of disaccharide fatty acid esters. I Am Oil Chem Soc 1994; 71 : 711-4.

27. GAO C, WHITCOMBE MJ, VULFSON EN. Enzymatic synthesis of dimeric and trimeric sugar fatty acid esters. Enz Microb Tech $1999 ; 25$ : 264-70.

28. GRIDLEY J], HACKING AJ, OSBORN HMI, SPACKMAN DG. Regioselective lipase-catalyzed acylation of 4,6-O benzylidene- alpha and beta -D- pyranoside derivatives displaying a range of anomeric substituents. Tetrahedron 1998 ; 54 : 14925-46.

29. REDMANN I, PINA M, GUYOT B, BLAISE P, FARINES M, GRAILLE J. Chemoenzymatic synthesis of glucose fatty esters. Carb Res $1997 ; 300$ : 103-8.

30. SCHLOTTERBECK A, LANG A, WRAY V, WAGNER F. Lipase-catalyzed monoacylation of fructose. Biotechnol Lett $1993 ; 15: 61-4$.

31. GBEKELOLUWA B, OGUNTIMEIN B, ERDMANN H, SCHMIDT RD. Lipase catalysed synthesis of sugar ester in organic solvents. Biotechnol Lett 1993; $15: 175-80$.

32. CLIFFORD MN. Chlorogenic Acids. In : Clark RJ, Macrae R, eds. Coffe Voll Chemistry. London : Elsevier Applied Science, 1985 : 153-202.

33. MARUTA Y, KAWABATA J, NIKI R. Antioxidative caffeoylquinic acid derivatives in the roots of burdock. / Agric Food Chem $1995 ; 43$ : 2592-6.

34. SCHOLTZE, HEINRICH M, HUUNKLER D. Caffeoylquinic acids and some biological activities of Pluchea symphytiflia. Planta Med $1994 ; 60: 360-4$.

35. GUYOT B, BOSQUETTE B, PINA M, GRAILLE J. Esterification of phenolic acids from green coffee with an immobilized lipase from Candida antarctica in solvent-free medium. Biotechnol Lett 1997 ; 19 : 529-32.

36. GUYOT B, GUEULE D, PINA M, GRAILLE J, FARINES V, FARINES M. Enzymatic synthesis of fatty esters in 5-caffeoyl quinic acid. Eur / Sci Technol 2000; 102 : 93-6.

37. BUISMAN GIH, VAN HELTEREN CTW, KRAMER GFH, VELDSINK IW, DERKSEN JTP, CUPERUS FP. Enzymatic esterifications of functionalized phenols for the synthesis of lipophilic antioxidants. Biotechnol Lett $1998 ; 20$ : 131-6.

38. PRIYA K, CHADHA A. Synthesis of hydrocinnamic esters by Pseudomonas cepacia lipase. Enz Microb Technol $2003 ; 32$ : 485-90.

39. STAMATIS H, SERETI V, KOLISIS FN. Studies on the enzymatic synthesis of lipophilic derivatives of natural antioxidants. / Am Oil Chem Soc 1999; 76 : $1505-10$

40. COMPTON DL, LASZLO JA, BERHOW MA. Lipase-catalyzed synthesis of ferulate esters. J Am Oil Chem Soc $2000 ; 77$ : 513-9.
41. SABALLY K, KARBOUNE S, SAINT LOUIS R, KERMASHA S. Lipase-catalyzed transesterification of trilinolein or trilinolenin with selected phenolic acids. I Am Oil Chem Soc 2006 ; 83 : 101-7.

42. SABALLY K, KARBOUNE S, SAINT LOUIS R, KERMASHA S. Lipase-catalyzed transesterification of dihydrocaffeic acid with flaxseed oil for the synthesis of phenolic lipids. J Biotechnol 2006 ; 127 : 167-76.

43. KANDASWAMI C, MIDDLETON JR. E. Flavonoids as antioxidants. In : Shahidi F, ed. Natural antioxidants: Chemistry, Health effects and applications. Illinois, USA : AOCS press, Campaign, 1997 : 174-203.

44. KONTOGIANNI A, SKOURIDOU V, SERETIV, STAMATIS H, KOLISIS FN. Regioselective acylation of flavonoids catalyzed by lipase in low toxicity media. Eur J Lipid Sci Technol 2001 ; 103 : 665-70.

45. KONTOGIANNIA, SKOUDIROU V, SERETIV, STAMATIS H, KOLISIS FN. Lipase-catalyzed esterification of rutin and naringin with fatty acids of medium carbon chain. J Molec Cat B Enz 2003 ; 21 : 59-62.

46. GAYOT S, SANTARELLIX, COULON D. Modification of flavonoid using lipase in non-conventional media : effect of the water content. / Biotechnol $2003 ; 101: 29-36$.

47. FRANKEL EN, HUANG S-W, AESCHBACH R, PRIOR E. Antioxidant activity of a rosmary extract and its constituents, carnosic acid, carnosol and rosmarinic acid, in bulk oil and oil-in-water emulsion. J Agric Food Chem 1996; 44 : 131-5.

48. HUANG S-W, FRANKEL EN, SCHWARZ K, AESCHBACH R, GERMAN JB. Antioxidant activity of carnosic acid and methyl carnosate in bulk oils and oil-in-water emulsions. J Agric Food Chem 1996 ; 44 : 2951-6.

49. PRYOR WA, STRICKLAND T, CHURCH DF. Comparison of the efficiencies of several natural and synthetic antioxidants in aqueous sodium dodecyl sulphate micelle solutions. / Am Chem Soc 1988 ; 110 : 2224-9.

50. HUANG S-W, HOPIA A, SCHWARZ K, FRANKEL EN, GERMAN JB. Antioxidant activity of $\alpha$-tocopherol and Trolox in different lipid substrates : bulk oils vs. oil-in-water emulsions. J Agric Food Chem 1996 ; 44 : 444-52.

51. FRANKEL EN, HUANG S-W, KANNER J, GERMAN JB. Interfacial phenomena in the evaluation of antioxidants : bulk oil vs. emulsions. J Agric Food Chem $1994 ; 42$ : 1054-9.

52. PORTER WL. Paradoxical behavior of antioxidants in food and biological systems. Toxicol Ind Health 1993 ; 9 : 93-122.

53. FIGUEROA-ESPINOZA MC, VILLENEUVE P. Phenolic acids enzymatic lipophilization. J Agric Food Chem $2005 ; 53$ : 2779-87.

54. SABALLY K, KARBOUNE S, YEBAOH FK, KERMASHA S. Enzymatic esterification of dihydrocaffeic acid with linoleyl alcohol in organic solvent media. Biocat Biotrans $2005 ; 23: 37-44$.

55. SABALLY K, KARBOUNE S, SAINT LOUIS R, KERMASHA S. Lipase-catalyzed esterification of linolenyl alcohol with selected phenolic acids in organic solvent media. Appl Biochem Biotechnol 2005 ; 127 : 17-28.

56. ANSELMI C, CENTINI M, GRANATA P, et al. Antioxidant activity of ferulic acid alkyl esters in a heterophasic system : a mechanistic insight. J Agric Food Chem $2004 ; 52: 6425-32$.

57. LEY JP, BERTRAM HJ. 3,4-Dihydroxymandelic acid amides of alkylamines as antioxidants for lipids. Eur / Lipid Sci Technol 2003 ; 105 : 529-35.

58. TRUJILLO M, MATEOS R, TERAN LC, et al. Lipophilic hydroxytyrosyl esters. Antioxidant activity in lipid matrices and biological systems. J Agric Food Chem $2006 ; 54: 3779-85$.

59. MELLOU F, LAZARI D, SKALTSA H, TSELEPIS AD, KOLISIS FN, STAMATIS H. Biocatalytic preparation of acylated derivatives of flavonoid glycosides enhances their antioxidant and antimicrobial activity. J Biotechnol 2005 ; $116: 295-304$. 